Maria Cecília deS. Minayo ${ }^{1}$

Edinilsa R. de Souza 1

Simone G. de Assis 1

Otávio Cruz Neto 1

Suely F. Deslandes 1

Cosme M. F. P. da Silva 1

\section{Avaliação dos Centros de O rientação e Apoio Sorológico/CTA/Coas da Região Nordeste do Brasil}

\author{
Evaluation of $O$ rientation and Serological \\ Support (Anonymous HIV Testing) Centers \\ in the Brazilian Northeast
}

1 Centro Latino Americano de Estudos deViolência e Saúde “Jorge Careli", Escola Nacional de Saúde Pública, Fundação Oswaldo Cruz. Av. Brasil 4036, sala 702 Rio de Janeiro, RJ 21040-361, Brasil.
Abstract This article presents the results of an evaluation of Orientation and Serological Support Centers, or Anonymous HIV Testing Centers, in the Northeast of Brazil. Methodological triangulation was used to eval uate these health programs and services, including qualitative and quantitative methodology and pointing to the insufficiency of single-discipline reasoning to encompass phenomena in social organizations. The article al so shows results from this triangulation experience, seeking to combine Social Sci ences and Epidemiology. It describes the structure and dynamics of these services, analyzes the relationships, perceptions, and representations of the actors, presents a self-evaluation by the professionals, expounds on some quantitative results, and discusses somelimits and problems, as well as proposals to overcomethem.

Key words Program Evaluation; Health Services Research; Research on Health Personnel; Community Health Services; Acqui red Immunodeficiency Syndrome

Resumo Este artigo apresenta os resultados da aval iação dos Centros de Orientação e Apoio Sorológico/CTA/Coas do Nordeste do País. Para avaliar esses programas/serviços desaúde, utilizouse a triangulação de métodos, que consiste na arti culação das abordagens quantitativa e qual itati va,mostrando a insufi ciência da razão uni disci pl inar para abranger os fenômenos contidos numa organi zação social. São também apresentados os resultados dessa experiência de triangulação, que busca integrar a epidemiologia e as ciênci as sociais. Mostra a estrutura formal e dinâmica dos serviços investi gados, anal isa as relações, percepções e representações do conjunto de atores, efetua uma auto-avaliação dos profissi onais, demonstra al guns resultados quantitati vos de sua atuação e apresenta os principais limites, problemas e propostas de superação. Palavras-chave Avaliação de Programas; Pesquisa sobre Serviços de Saúde; Pesquisa sobre Recursos Humanos de Saúde; Serviços de Saúde Comunitária; Síndrome de Imunodeficiência Adquirida 
Introdução

No início da década de 90, a Coordenação do Programa Nacional de Doenças Sexualmente Transmissíveis (DST)/Aids do Ministério da Saúde, em conjunto com as secretarias estaduais e municipais de saúde, criou unidades públicas de saúde em todo o país, os CTA (Centros de Testagem Anônima)/ Coas (Centros de Orientação e Apoio Sorológico), com as seguintes finalidades: oferecer testagem anônima para o vírus da Aids e, ao mesmo tempo, desenvolver um processo de prevenção e orientação aos seus usuários; possibilitar a realização de testes sorológicos para infecção pelo HIV na própria rede pública; diminuir a demanda de procura por outros serviços de diagnóstico da infeção pelo HIV (como os hemocentros) e tornar esse serviço acessível à população sem condições de procurar os laboratórios particulares. Uma vez implantados, esses Centros passaram a funcionar sem que uma avaliação sistemática tivesse sido realizada.

Este artigo tem como objetivo apresentar uma experiência, real izada em 1996, de avaliação dos 17 Centros de Orientação e Apoio Sorológico/CTA/Coas, situados na região Nordeste (NE) do Brasil: Bahia (Programa Estadual e Municipal de DST/Aids); Sergipe (Programa Estadual de DST/Aids de Aracaju); Alagoas (Programa Estadual e Municipal de DST/Aids de Maceió e o Programa Municipal de Arapiraca); Pernambuco (Programas Municipais de DST/ Aids Recife, Olinda e Caruaru); Paraíba (Programa Estadual de DST/ Aids de João Pessoa); Rio Grande do Norte (Programa Estadual de DST/ Aids de Natal); Ceará (Programas Estadual e Municipal de DST/Aids de Fortaleza); Piauí (Programas Estaduais de DST/Aids de Teresina e Parnaíba) e M aranhão (Programa Municipal de DST / Aids de São Luís e Programa Estadual de DST/ Aids de Imperatriz - Brasil, 1996).

A aval iação teve os seguintes objetivos: a) caracterizar as condições de funcionamento dos 17 CTA/Coas/ NE, em termos de recursos físicos, humanos e institucionais; b) descrever o processo de atendimento desenvolvido em cada um dos Centros (rotinas e relações); c) verificar a qualidade e o papel estratégico das informações geradas a partir do atendimento prestado; d) sistematizar e analisar os dados quantitativos produzidos pelos CTA/Coas; e) analisar as relações dos CTA/ Coas com a rede de serviço de atendimento às DST/ Aids; $f$ ) avaliar as percepções dos profissionais sobre o projeto CTA/ Coas, as condições de trabalho, as dificuldades encontradas na execução do projeto, as propostas de mudança e as perspecti- vas para o futuro; g) avaliar as percepções dos usuários sobre o serviço CTA/Coas, as relações com os profissionais, a efetividade do atendimento e a sua satisfação com a prática do aconselhamento. Naturalmente, não se dispõe neste artigo de espaço suficiente para tratar todos estes itens de forma integral, sendo, portanto, necessário o resumo dos principais aspectos abordados.

\section{Material e métodos}

O método adotado - avaliação por triangulação de métodos - integrou diferentes estratégias metodológicas: a) a análise das estruturas, dos processos e dos resultados, considerados os três pilares básicos da avaliação tradicional (Donabedian, 1980); b) a compreensão das relações entre todos os atores envolvidos na implementação das ações; c) a visão que esse conjunto de atores constrói sobre todo o projeto: seu desenvolvimento, as relações hierárquicas e técnicas, fazendo dele um constructo específico (Shutz, 1982; Schrimshaw \& Hurtado, 1987).

Além da integração obj etiva e subjetiva no processo avaliativo, a experiência incluiu os atores do programa não apenas como alvo analítico, mas também enquanto sujeitos de auto-avaliação, que, no desenrolar do processo, ap ropriam-se da compreensão dos dados quantitativos e qualitativos para as mudanças necessárias e o aprimoramento de suas ações.

A triangulação em sentido estrito é definida como a estratégia metodológica que abrange o objeto de investigação sob, pelo menos, três ângu los distintos que se articulam a partir da: 1) cooperação de distintos pesquisadores (discutindo, diferenciando e relacionando teorias, conceitos, noções e métodos, tratados como fragmentos teóricos de uma abordagem mais ampla), substituindo a hierarquia a priori dos campos científicos por uma visão cooperativa entre el es e o mundo da vida (Habermas, 1987); 2) integração de distintos métodos, permitindo o aprofundamento teórico-metodológico em relação ao conhecimento do objeto. É preciso ter clareza de que se trata de combinação, de triangulação de métodos que conservam sua especificidade no diálogo inter ou transdisciplinar, entendendo-se que se realiza aqui uma dialética entre integração e distinção (Kant, 1980; Minayo, 1992; Samaja, 1992). Defende-se que os estudos quantitativos e qualitativos não se opõem nem se contradizem, mas se complementam (Minayo \& Sanches, 1993); 3) integração coerente e criativa de distintas técnicas 
para a construção dos dados, seja no âmbito quantitativo, ou qualitativo.

A avaliação dos CTA/ Coas/ NE foi considerada com base em uma abordagem que buscou, portanto, articular ciências sociais e epidemiologia, analisando as questões contextuais, enriquecendo o marco teórico com temas interdisciplinares e pautando-se por uma concepção de pesquisa estratégica. Visou integrar a auto-avaliação dos atores envolvidos no programa, levando em conta a dinâmica das relações hierárquicas e a compreensão das estruturas enquanto construções históricas. Naturalmente, há que se ressaltar que, se a triangulação metodológica tem uma larga tradição nas ciências sociais (Denzin, 1970; Jick, 1979; André, 1982; Samaja, 1992), na área da saúde pública, ainda se constitui como proposta em construção. O presente trabalho reflete esse esforço e revela suas limitações quando, diante da precariedade concreta dos dados, recorre ao recurso descritivo, buscando integrá-lo numa dimensão reflexiva e crítica.

A equipe que realizou a avaliação foi composta por sociólogos, antropólogo, médico, psicólogo, epidemiologista, estatístico e técnicos da Escola Nacional de Saúde Pública (Ensp) Fundação Oswaldo Cruz (Fiocruz) - e por 17 profissionais de saúde dos CTA/ Coas avaliados. Estes foram treinados em relação ao projeto, à filosofia da avaliação, aos instrumentos de trabalho de campo, aos procedimentos e técnicas de abordagem de avaliação, numa perspectiva de transferência de tecnologia.

Como aspectos organizacionais, foram privilegiados: a estrutura dos serviços, o processo de trabalho, a equipe técnica multiprofissional, os dados neles produzidos, o processo de atendimento, a relação com a rede de serviços assistenciais (referência e contra-referência) ea relação dos profissionais com os usuários.

Do ponto de vista epidemiológi co, foram elaborados três instrumentos, preenchidos pelas equipes dos CTA/ Coas, nos quais foram solicitados: (a) um relatório descritivo contendo uma análi se crítica quanto à sistematicidade, suficiência e qualidade dos registros das atividades desenvolvidas pelos serviços; (b) um questionário a ser preenchido com dados que possibilitassem construir indicadores de produção e (c) um questionário para preenchimento das informações que permitissem traçar um perfil da demanda atendida.

Na abordagem qualitativa, foram utilizados dois roteiros de entrevistas semi-estruturadas e um roteiro de observação de campo.

Nas entrevistas, foram abordadas as percepções dos profissionais dos CTA/Coas sobre o proj eto CTA/ Coas (história, processo); as relações com o programa DST/Aids e com os usuários; as condições e processo de trabalho (rotinas e relações); as relações com a rede de saúde local; as dificuldades encontradas na execução do projeto; as propostas de mudança; as perspectivas para o futuro e acerca da possibilidade de realização dos princípios orientadores do CTA/ Coas.

No segundo roteiro de entrevistas dirigido ao usuário, foram contempladas suas percepções sobre o serviço CTA/ Coas, tendo como base seus princípios organizacionais; as relações com os profissionais; as relações com a rede de serviços de saúde; a efetividade do atendimento; a satisfação com a prática do aconselhamento e a importância da existência do CTA/ Coas.

Nas observações de campo, foram investigados itens como: condições de trabalho; relações entre profissionais e usuários; cumprimento dos princípios organizacionais.

$\mathrm{Na}$ avaliação, realizaram-se 41 entrevistas com profissionais dos CTA/Coas; 61 entrevistas com usuários; 17 relatórios de observação de campo; 17 documentos de auto-avaliação das equipes de cada CTA/ Coas e relatórios dos dados consolidados do atendimento dos 13 CTA/Coas já implantados.

Os dados apresentados a seguir foram construídos, por conseguinte, com base na leitura quantitativa e qualitativa desse acervo. Em razão da limitação de espaço, optou-se por sintetizarem-se neste artigo os resultados mais importantes.

O trabal ho de campo foi realizado em cada um dos CTA/Coas pela equipe do Centro Latino Americano de Estudos de Violência e Saúde "Jorge Careli" (Claves)/Ensp e pelos profissionais do serviço, mediante entrevistas, observação e análise documental.

\section{Resultados}

\section{1) Processo de implantação}

e rotina do atendimento

Os problemas mais relatados no processo de implantação dos CTA/ Coas foram: dificuldade em obter um local de fácil acesso para a população (quanto à facilidade de transporte público e ao elevado fluxo de pessoas); ausência de local adequado para sua instalação (instalações físicas com adequado número de salas, em bom estado de ventilação, mobiliadas, entre outras necessidades); precário número de profissionais selecionados, além do temor da 
população da área circundante quanto ao contato com pessoas portadoras do vírus HIV. Alguns profissionais de saúde também dificultaram a implantação dos CTA/Coas, discriminando e rejeitando a idéia de atuarem no atendimento a doentes com Aids ou portadores do HIV.

No que tange à formação da equipe eà capacitação dos profissionais dos CTA/ Coas, observou-se que a seleção dos mesmos foi feita ora a partir de indicação do coordenador do Programa de DST/ Aids do estado ou município, ora pelo interesse do próprio profissional. I nicialmente, alguns profissionais de nível superior foram treinados não só visando à atualização do conteúdo e das práticas de atendimento aos usuários (especialmente aos portadores do vírus HIV), como também para conhecerem as propostas do CTA/Coas. Esses, ao retornarem, repassaram o treinamento aos colegas que foram sendo incorporados. Os CTA/ Coas de São Paulo e Rio de Janeiro foram os principais locais de treinamento para os profissionais que atuam no Nordeste. Mais recentemente, o CTA/Coas de Aracaju passou a ser também um centro treinador da região.

A equipe dos 136 técnicos que atuam nos CTA/Coas possui um perfil composto em $57 \%$ por profissionais de nível superior: médicos, assistentes sociais, psicólogos, enfermeiros e farmacêuticos. Apenas seis médicos estão envolvidos no atendimento prestado pelos CTA/ Coas, e somente os CTA/ Coas de Pernambuco possuem uma equipe multiprofissional completa. Em relação aos técnicos de nível médio, observa-se que as figuras do recepcionista e auxiliares/técnicos de enfermagem são importantes, pois são eles os responsáveis pela acoIhida aos usuários e pela coleta de material para exame.

Com rel ação aos turnos de trabalho, notase que, em 12 dos 17 CTA/Coas, o atendimento é feito em horário parcial. Apenas cinco oferecem atendimento durante todo o dia. O reduzido turno de trabalho deve-se a dois fatores: baixa demanda do serviço e pequeno número de profissionais envolvidos, dispondo de pouca carga horária no serviço.

No âmbito da rotina de atendimento, o trabalho com o usuário tem início na recepção (conversa inicial, preenchimento do questionário, pré-teste e identificação por uma senha visando ao sigilo e à confidencialidade). O passo seguinte é o aconsel hamento coletivo, seguido do individual (opcional). Nesse momento, o usuário opta ou por sair do sistema, ou pela realização do teste. A seguir dá-se a coleta do material e o agendamento para a entrega do resultado. Esta entrega é feita com aconselhamento individual, momento em que o usuário com resultado negativo recebe novamente orientação para manter os cuidados necessários à prevenção das DST/ Aids, ganhando, em alguns CTA/Coas, preservativos. A pessoa com resultado positivo conta com um primeiro apoio emocional, orientações, preservativos e é encaminhada para a rede pública de saúde. Essa dinâmica pode ser observada na Figura 1.

Embora não tenha sido incluída no fluxograma, a distribuição de preservativos foi incorporada à pratica da maioria dos CTA/Coas.

A rotina do aconselhamento coletivo prevê a abordagem dos seguintes tópicos: explicação sobre o CTA/Coas; informações gerais sobre modos de transmissão; prevenção; janela imunológica e testes. $\mathrm{O}$ aconselhamento coletivo enfatiza as questões ligadas à Aids, mas também são enfocadas as DSTs. Essa atividade é desenvolvida em forma de palestra, com duração variável de vinte minutos a uma hora. Trata-se de um encontro informativo visando fornecer ao usuário um patamar mínimo de esclarecimento para respaldar suas decisões e esclarecer suas dúvidas. A condução da reunião, bem como a interação com os usuários, depende do nível de formação/informação do conseIheiro, sua criatividade e grau de envolvimento com a atividade. Neste processo, é fundamental haver o entrosamento entre os membros da equipe; a discussão sobre o desempenho de cada profissional; o apoio psicológico aos membros da equipe; atualização permanente; motivação social e profissional; material didático pedagógico adequado e ambiente físico apropriado.

Outro ponto a ser destacado é o da coleta e realização dos exames para HIV. Alguns CTA/ Coas apresentam dificuldades nessa tarefa, comprometendo o atendimento prestado aos usuários. Os principais fatores que influenciam a qualidade desse atendimento estão ligados à proximidade do CTA/ Coas ao laboratório que realizará os exames e à demanda excessiva por exames de diversos tipos que chegam a este último.

A questão da referência e contra-referência para os portadores do vírus da Aids mostrou-se muito desgastante na maioria das cidades. 0 reduzido número de leitos hospitalares e a precariedade dos serviços ambulatoriais com enfoque multiprofissional são responsáveis por essa situação. A rede de referência é composta por hospitais públicos em dez dos 17 municípios avaliados. Só cinco CTA/ Coas contam com unidades ambulatoriais especializadas no atendimento a DST/Aids, para as quais referenciam 
Fluxograma de atendimento dos Centros de Testagem Anônima/Centros de Orientação e Apoio Soroló gico da região Nordeste, Brasil.

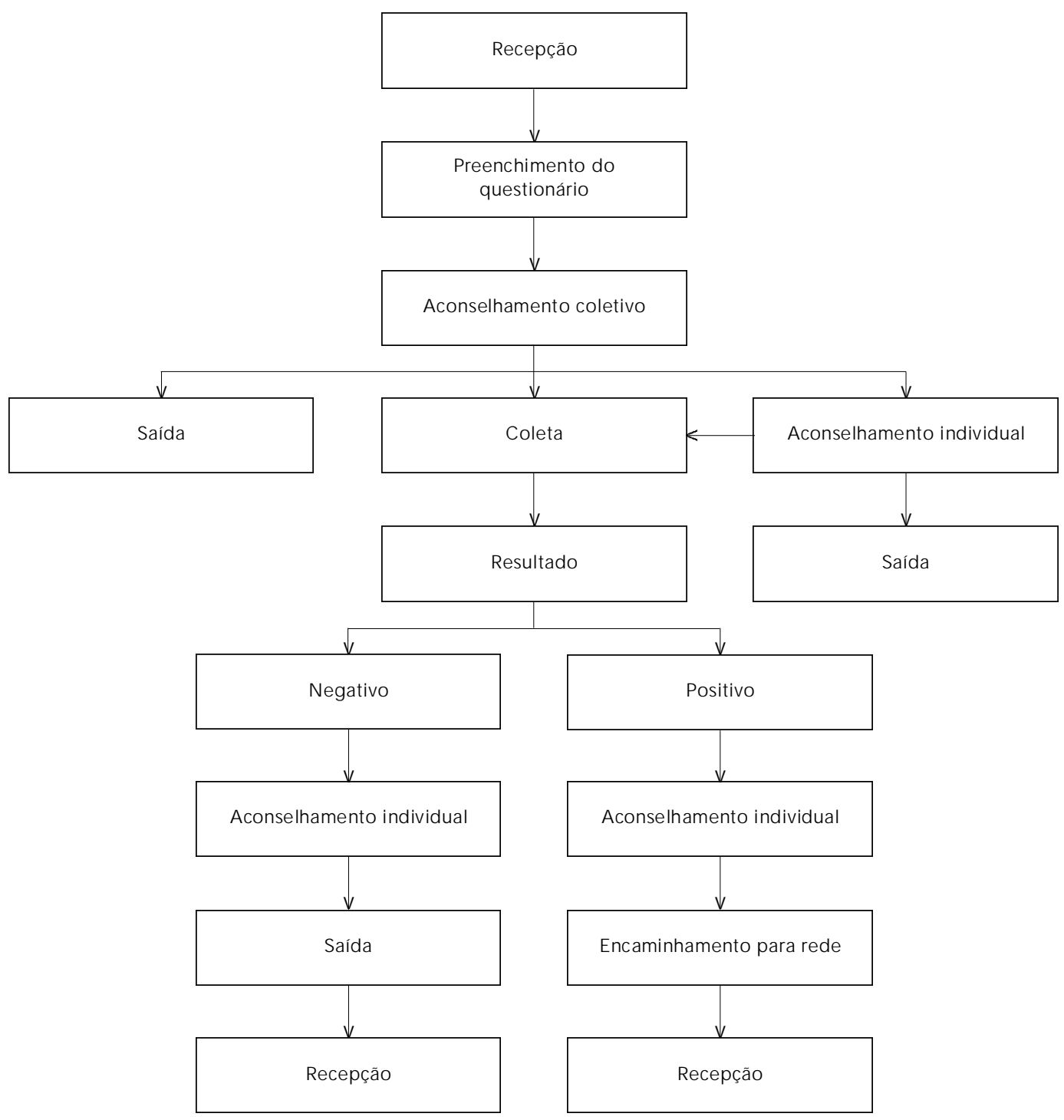

os casos positivos. A contra-referência foi citada apenas por quatro CTA/Coas. Ela ocorre informalmente, sendo decorrente da boa vontade dos profissionais da rede. A informação formalizada existia em apenas duas unidades.

Embora a diretriz dos CTA/ Coas preconize o encaminhamento dos portadores de HIV para a rede de saúde, o profissional deste serviço se vê em conflito, pois alguns CTA/Coas não fornecem cópia do resultado para os usuários, e os que o fazem - em razão do si gi lo - entregam somente o resultado anônimo, que não é aceito pela rede. Tal procedimento obriga os pacientes a refazerem o teste, retardando o início do tratamento. Desta forma, al guns usuários já infectados acabam voltando para o CTA/Coas em busca de atendimento, o que não é sua finalidade.

A análise efetuada com base nos instrumentos utilizados pelos CTA/ Coas reafirma as dificuldades na referência e contra-referência. Nessa análise, observou-se que apenas seis dos 13 CTA/Coas já implantados conseguem informar se o usuário foi encaminhado aos serviços 
de referência por ser identificado como HIV+ ou por outras DST. O processo de avaliação gerou a necessidade de que as equipes identificassem os encaminhamentos explicitando os casos de Aids e de outras doenças transmissíveis separadamente. Essa rotina passou a ser feita no CTA/Coas de Olinda, após o treinamento realizado para a pesquisa.

Observou-se que, mesmo quando encaminhados pelo CTA/Coas, nem todos os usuários eram atendidos. É bastante preocupante o fato de que $12 \%$ dos usuários com teste positivo para HIV eque $2 \%$ daqueles com teste positivo para outras DST deixem de ser atendido na rede.

O financiamento essencial aos Projetos CTA/Coas é fruto do interesse local e de convênio entre as secretarias de saúde (estadual ou municipal) e o Ministério da Saúde, por intermédio da Coordenação Nacional de DST/Aids. A responsabilidade das secretarias na construção, reforma e manutenção do espaço físico, assim como no que se refere ao pagamento de recursos humanos e à compra do material de consumo, é compartilhada com o Ministério da Saúde; contudo, a ênfase para a continuidade desses serviços vem recaindo sobre os estados e municípios. Apesar de o Ministério da Saúde ter consi derado a questão das parcerias como o fundamento básico para a estruturação e funcionamento dos CTA/Coas, o que se percebe, na realidade, é que algumas questões burocráticas vêm dificultando a concretização desse trabalho conjunto. Entre os principais problemas percebidos nesta aval iação estão a demora na elaboração do convênio, o atraso no repasse dos recursos para os estados e municípios, as dificuldades locais na execução do próprio orçamento e a ausência de uma rede de informação mais ágil, interligando os técnicos dos CTA/ Coas com os respectivos setores administrativos.

2) Percepções e o pinião do usuário sobre o CTA/Coas

Val endo-se das entrevistas realizadas com os usuários, pode-se perceber que, em relação ao conhecimento sobre os serviços CTA/ Coas, a divulgação pela rede de serviços públicos de saúde foi uma das principais fontes de informação, tão citada quanto a rede pessoal de informações (divulgação entre amigos ou parentes que já haviam utilizado o serviço anteriormente). Foram vários os motivos que levaram os usuários a procurar o CTA/Coas. Uma única relação ou prática considerada de risco pelo usuário pode fazer com que ele, anos depois, busque a realização do teste. A desconfiança sobre o comportamento sexual do parceiro foi muito citada por mulheres como o principal motivo para procurar o CTA/ Coas. Essa constatação coincide com os depoimentos dos profissionais de que o principal meio de divulgação do seu trabalho são as pessoas que compareceram ao serviço, gostaram do atendimento e trouxeram outras pessoas. Curiosamente, as ONGs têm participação inexpressiva na divulgação, o que aponta para a necessidade de um trabal ho de sensibilização desses agentes para a divulgação e encaminhamento dos usuários.

A maioria dos entrevistados vinha pela primeira vez ao CTA/ Coas. Aqueles que retornavam faziam-no por diferentes motivos: estar em janela imunológica; pegar camisinha; repetir o teste; ter tido alguma nova situação de exposição; saber resultado e receber apoio emocional dos orientadores. Pessoas cujos testes foram positivos declararam ter gostado de conversar com os profissionais do CTA/Coas, pois sentiam-se amparadas nos momentos de depressão. A espontânea continuidade de vínculo revela a necessidade ou carência desse tipo de assistência no serviço ambulatorial e hospitalar. O sistemático retorno de usuários para receber preservativos também indica a possibilidade de uma orientação mais permanente, uma vez que se estabelece um elo propício à prevenção de novos riscos.

Quanto às percepções do usuário sobre os objetivos do CTA/Coas, abre-se um amplo campo discursivo que abrange os múltiplos aspectos simbólicos envolvidos, tanto nas formas de disseminação do HIV, como nas perspectivas sociais de controle e prevenção. Por outro lado, indicam como os usuários decodificam a orientação produzi da em cada CTA/ Coas: alguns o vêem como um serviço com papel humanitário, cuja atuação transcende as ações cotidianamente empreendidas. Esse sentimento foi insistentemente referido pela maioria dos entrevistados. Medo de receber um resultado positivo, receio de encontrar algum conhecido, vergonha/medo de entrar no serviço e ser taxado de doente ou homossexual, de sentir-se como provável portador: essas vivências dão lugar a uma sensação de confiança e tranqüilidade após o atendimento; todavia, tornam-se novamente intensas quando o resultado atrasa ou é inconclusivo.

A receptividade e o tratamento dispensado pelos profissionais foram extremamente elogiados pelos usuários, sendo a relação usuárioprofissional vista como amigável e solidária. Esses valores de amabilidade e respeito são reconhecidos como fatores que distinguem o CTA/Coas perante o julgamento de seus usuá- 
rios. O nível de embrutecimento das relações no dia-a-dia e a impessoalidade que rege o atendimento nos serviços públicos de saúde fazem com que o usuário do CTA/ Coas afirme: "nem pareceservi ço público".

$\mathrm{O}$ aconsel hamento foi considerado eficaz para tirar dúvidas e trazer informações até então desconhecidas, e o uso de recursos visuais foi bastante valorizado pelos usuários. O constrangimento inicial em revelar aspectos da intimidade é parcialmente vencido quando o usuário percebe segurança e profissionalismo do conselheiro.

Esta delicada relação precisa ser preservada em detrimento da falta de tempo e do formalismo impessoal; exige, portanto, um trabaIho constante de atualização. O nível de confiança que é depositado pelos usuários é imenso; é grande também o interesse destes nas informações.

3) Qualidade dos instrumentos utilizados pelos CTA/Coas

A análise crítica dos formulários usados pelos serviços mostrou que há CTA/Coas que utilizam muitos instrumentos, tendo sido referido pelos próprios profissionais que tais formulários contêm informações repetidas. Por outro lado, há outros CTA/ Coas carentes de informações, pois os poucos instrumentos utilizados não os suprem com os dados necessários para que tenham um perfil da sua demanda e das características epidemiológicas dos usuários. Outra observação importante é que os instrumentos não são padronizados; muitas vezes uma mesma informação aparece de maneira completamente diferente nos questionários, em termos de linguagem e pontos de corte ou faixas/ interval os de classificação para as variáveis investigadas.

É importante destacar que a grande mai oria dos CTA/Coas não efetua a análise dos dados do seu atendimento. Essa tarefa recai sobre as coordenações de epidemi ologia das secretarias municipais e estaduais de saúde. Por isso, percebe-se por parte da equipe uma informação puramente empírica acerca da clientela atendida. Essa descon exão entre a execução do atendimento e a consolidação dos dados gerados por el e leva ao desconhecimento das características básicas da clientela atendida e cria desinteresse em torno do processo de geração, construção e disseminação da informação produzida no serviço.

A não-consolidação desses dados nos serviços é responsável por dificuldades de várias ordens que interferiram na construção dos in- dicadores quantitativos. Tornou-se impossível, nesta avaliação, a constituição de determinados indicadores gl obais para a região em estudo, tendo em vista que al guns dados não são coletados, enquanto outros o são de maneiras completamente diferentes, impedindo a comparação entre os CTA/ Coas. O período solicitado para a presente investigação (primeiro semestre de 1996 - janeiro a junho) nem sempre pôde ser assim considerado. Alguns CTA/Coas tinham sido implantados há menos tempo; em outras situações, a equipe consolidou os dados para um período diferente. A precária qualidade da informação levou à exclusão de alguns dados de determinados CTA/Coas. Desse modo, considerando-se os problemas mencionados, os indicadores aqui apresentados precisam ser relativizados enquanto expressão da realidade da região Nordeste.

Apesar das dificuldades relatadas, é preciso reconhecer que esta foi, para a maioria dos CTA/Coas, a primeira tentativa de consolidação dos dados do seu aten dimento, tarefa extremamente difícil para al gumas equipes que não possuíam os necessários conhecimentos básicos de estatística e/ ou epidemiologia. Entretanto, a pesquisa foi considerada parte de um processo de avaliação mais amplo, que não se limita à investigação efetuada, mas que terá continuidade nos CTA/ Coas, mediante modificações em suas rotinas de trabalho, inclusão de determinadas informações e adoção de certos instrumentos antes não utilizados. Neste sentido, caso se deseje que as equipes possam se realimentar com as informações geradas nos seus serviços e não continuem desconhecendo os dados neles produzidos, devem-se encarar as dificul dades e limites no manuseio quantitativo dos instrumentos, bem como a análise dos dados nestes contidos, como mais um dado extraído dessa avaliação, na medida em que apontam para a necessidade de homogeneização dos instrumentos e treinamento das equipes.

\section{4) Análise dos dados gerados nos CTA/Coas}

No que se refere à construção do perfil da clientela dos CTA/Coas/NE, observou-se que $54 \%$ dos usuários eram do sexo masculino e que $57 \%$ tinham até 29 anos, constituindo uma população jovem e natural do próprio município (77\%) que procura esses serviços. Maiores freqüências foram observadas nas faixas de 20 a 24 anos (23\%) e de 25 a 29 anos (22\%). A grande maioria del es era sol teira (59\%), embora os casados/amasiados tenham representado $29 \%$. O nível de escolaridade em $72 \%$ deles si- 
tuava-se entre o primeiro (39\%) e o segundo (33\%) graus.

A informação sobre a renda dos usuários ficou prejudicada, pois essa variável é classificada de modo muito heterogêneo entre os CTA/Coas. Contudo, considerando como tendo baixa renda aqueles que não possuíam nenhuma renda ou cuja renda era de até três salários mínimos, observou-se que cerca de $80 \%$ dos usuários se concentram nesta categoria.

A cor da pele dos usuários foi informada por apenas quatro dos CTA/Coas, e sua análise também deve ser relativizada. I soladamente, a cor branca apareceu como a mais freqüente (38\%), embora os pardos e negros tenham constituído, juntos, a maior parcela da clientela dos CTA/Coas que informaram: (49\%).

Alguns indicadores de processo de atendimento podem ser verificados nas Tabelas 1 a 5 a seguir. Antes, porém, é preciso esclarecer que os CTA/Coas de Parnaíba - PI ; João Pessoa PB; Caruaru - PE e o da Secretaria Municipal de Saúde de Salvador foram excluídos das tabelas porque se encontravam em fase de implantação à época da pesquisa.

Na Tabela 1, percebe-se que as médias diárias tanto dos aconselhamentos, quanto dos testes realizados variam entre os CTA/Coas da Região. Observa-se que os CTA/ Coas de Aracaju (Secretaria Estadual de Saúde/SES), Maceió (Secretaria Municipal de Saúde/ SMS), São Luís
(SMS) e Fortaleza (SES) apresentam as maiores médias diárias, enquanto os CTA/ Coas de Imperatriz (SES), Natal (SES) e Arapiraca (SMS) apresentam as menores médias.

Dos 9.814 usuários que procuraram os CTA/Coas/NE no período investigado, $85 \%$ efetuaram os testes. Como se verifica na Tabela 2, esse valor variou entre 66\% (em Aracaju) e $100 \%$ em Maceió. O índice total de positividade foi de $4,4 \%$, isto é, foram encontrados 431 testes positivos em relação aos 9.758 realizados na região em estudo. Ainda na Tabela 2, verifica-se que o menor índice encontrado deu-se no CTA/Coas municipal de Maceió (1\%), e o maior, no estadual de Imperatriz (7,4\%). Os homens apresentaram uma maior proporção de positi vidade para o teste na mai oria dos CTA/ Coas: para o total da região, observou-se uma proporção de 2,4 homens infectados para cada mulher. Ao todo, os CTA/Coas identificaram 305 homens infectados entre os 5.370 que fizeram o teste, e 125 mulheres infectadas entre as 4.344 que também fizeram o teste. Deve-se considerar que os dados desta tabela excluem a informação dos CTA/ Coas estadual de Fortaleza (informação deficiente) e apresentam um período distinto para o CTA/Coas Estadual de Salvador: de outubro de 1994 a jun ho de 1996.

$\mathrm{Na}$ Tabela 3 encontram-se as proporções com que cada categoria de exposição contribuiu no total de testes realizados.

Tabela 1

N úmero de aconselhamentos e testes realizados e respectivas médias em Centros de Testagem Anônima/

Centro de O rientação e Apoio Sorológico da região Nordeste, Brasil.

\begin{tabular}{|c|c|c|c|c|c|c|}
\hline CTA/Coas & Período & Funcionamento & Aconselhamentos & Média diária & Testes realizados & Média diária \\
\hline Imperatriz (SES*) & 09/04/96 a 30/06/96 & 5 dias por semana & 166 & 3,3 & 162 & 2,9 \\
\hline São Luís (SM S**) & $01 / 96$ a $06 / 96$ & 5 dias por semana & 1.245 & 10,4 & 1.217 & 10,1 \\
\hline Teresina (SES) & $01 / 96$ a $06 / 96$ & 5 tardes por semana & 538 & 4,5 & 435 & 3,6 \\
\hline Fortaleza (SES) & $01 / 96$ a $06 / 96$ & 5 dias por semana & NP & NP & 1.429 & 11,9 \\
\hline Fortaleza (SMS) & $17 / 06 / 96$ a $30 / 08 / 96$ & 5 dias por semana & 420 & 8,4 & 392 & 7,8 \\
\hline Natal (SES) & $07 / 95$ a $09 / 96$ & 5 tardes por semana & 411 & 1,4 & 334 & 1,1 \\
\hline Olinda (SM S) & $01 / 96$ a $06 / 96$ & 5 dias por semana & 468 & 3,9 & 400 & 3,3 \\
\hline Recife (SMS) & $01 / 96$ a $06 / 96$ & 5 dias por semana & 1.225 & 10,2 & 1026 & 8,6 \\
\hline Aracaju (SES) & $01 / 96$ a $06 / 96$ & 5 tardes por semana & 975 & 8,1 & 648 & 5,4 \\
\hline Arapiraca (SMS) & $07 / 96$ a $08 / 96$ & 3ạ e 4a à tarde & 27 & 0,7 & 24 & 0,6 \\
\hline Maceió (SES) & $\mathrm{NI}$ & 3ạ, 4ạ e 5ạ pela manhã & 44 & - & 44 & - \\
\hline Maceió (SMS) & $01 / 96$ a $06 / 96$ & 2ạ e 5a pela manhã & 616 & 5,1 & 616 & 5,1 \\
\hline Salvador1 (SES) & $10 / 94$ a $06 / 96$ & 5 dias por semana & 3.679 & 8,8 & 3.031 & 7,2 \\
\hline Total & - & - & 9.814 & - & 9.758 & - \\
\hline
\end{tabular}

* Secretaria Estadual de Saúde; ** Secretaria Municipal de Saúde.

10 CTA/Coas Salvador (SES) apresento u um total de 1.199 aconselhamentos no período de 01/96 a 06/96, porém não informou o total de exames neste mesmo período, razão pela qual foi utilizado o total observado nesta tabela para o perío do de 10/94 a 06/96.

Obs.: NI = não informou; NP = não possui a informação. 
Proporção de testes realizados segundo a procura pelo serviço, e proporções de testes positivos em relação aos realizados segundo sexo. Centros de Testagem Anônima/Centro de Orientação e Apoio Sorológico da região Nordeste, Brasil.

\begin{tabular}{|c|c|c|c|c|}
\hline & \multicolumn{4}{|c|}{ Indicadores } \\
\hline & $\begin{array}{l}\mathrm{N} \circ \text { testes feitos } / \mathrm{n}^{\circ} \text { pessoas } \\
\text { que foram ao CTA/Coas }\end{array}$ & $\begin{array}{l}\text { Resultados + em relação ao } \\
\text { total de testes realizados }\end{array}$ & $\begin{array}{l}\mathrm{N} 0 \text { de testes }+ \text { em homens/ } \\
\text { total de homens que } \\
\text { fizeram o teste }\end{array}$ & $\begin{array}{l}\mathrm{N} 0 \text { de testes }+ \text { em mulheres/ } \\
\text { total de mulheres que } \\
\text { fizeram o teste }\end{array}$ \\
\hline Imperatriz (SES*) & $98 \%$ & 7,4 & 9,6 & 4,4 \\
\hline São Luís (SM S**) & $98 \%$ & 4,4 & 4,5 & 4,2 \\
\hline Teresina (SES) & $81 \%$ & 9,2 & 10,3 & 8,0 \\
\hline Fortaleza (SES) & NP & 2,8 & 5,4 & 0,5 \\
\hline Fortaleza (SMS) & $93 \%$ & 1,5 & 2,8 & - \\
\hline N atal (SES) & $81 \%$ & 2,4 & 2,4 & 2,5 \\
\hline Olinda (SM S) & $85 \%$ & 3,5 & 5,6 & 1,5 \\
\hline Recife (SMS) & $84 \%$ & 3,9 & 5,7 & 0,8 \\
\hline Aracaju (SES) & $66 \%$ & 2,0 & 2,2 & 1,7 \\
\hline Maceió (SES) & $100 \%$ & 2,3 & $\mathrm{NI}$ & $\mathrm{NI}$ \\
\hline Maceió (SMS) & $100 \%$ & 1,0 & 1,7 & 0,5 \\
\hline Salvador (SES) & $82 \%$ & 6,5 & 7,5 & 5,2 \\
\hline Total & $85 \%$ & 4,4 & 5,7 & 2,9 \\
\hline
\end{tabular}

* Secretaria Estadual de Saúde; ** Secretaria Municipal de Saúde.

Obs.: NI = não informou; NP = não possui a informação.

O CTA/C oas Arapiraca (SMS) informou apenas a proporção de $89 \%$ de testes

feitos em relação ao número de pessoas que foram a este serviço.

Tabela 3

Proporção de testes por categorias de exposição em relação ao total de testes realizados. Centros de Testagem Anônima/ Centro de Orientação e Apoio Sorológico da região Nordeste, Brasil.

\begin{tabular}{|c|c|c|c|c|c|c|c|c|}
\hline & \multicolumn{8}{|c|}{ Categorias de exposição } \\
\hline & Perinatal & Transfundido & Hemofílico & $\begin{array}{l}\text { Usuário } \\
\text { de droga } \\
\text { endovenosa }\end{array}$ & Homossexual & Bissexual & Heterossexual & Ignorado \\
\hline Imperatriz (SES*)1 & 1,7 & 4,1 & - & 1,7 & 1,2 & 11,6 & 79,6 & - \\
\hline São Luís (SM S**) & 0,2 & 0,7 & - & 0,2 & 2,5 & 6,0 & 55,9 & 34,6 \\
\hline Teresina (SES)1 & 1,8 & 1,1 & - & 4,3 & 5,4 & 10,1 & 76,5 & 1,8 \\
\hline Fortaleza (SES) & NP & 1,7 & - & - & 9,2 & 0,1 & 88,6 & 0,5 \\
\hline Fortaleza (SMS) & - & 4,3 & - & 0,3 & 6,4 & 8,7 & 80,4 & - \\
\hline Recife (SMS) & - & - & - & - & 10,4 & 5,2 & 44,2 & 40,2 \\
\hline Aracaju (SES) & 0,8 & 0,1 & - & 1,5 & 5,9 & 4,2 & 87,5 & - \\
\hline Arapiraca (SMS) & - & - & - & 4,2 & 12,5 & 4,2 & 75,0 & 4,2 \\
\hline Maceió (SMS) & - & - & - & - & 8,4 & 6,2 & 84,4 & 1,0 \\
\hline Salvador (SES) & - & - & - & - & 8,8 & 8,3 & 82,8 & - \\
\hline Total & 0,3 & 0,9 & - & 0,5 & 7,2 & 5,3 & 73,2 & 12,6 \\
\hline
\end{tabular}

1 Dez usuários em Imperatriz e nove em Teresina pertenciam a mais de uma categoria.

* Secretaria Estadual de Saúde; ** Secretaria Municipal de Saúde; N P = N ão possui a informação.

Obs.: CTA/Coas Olinda (SMS) e Maceió (SES) não informaram.

CTA/Coas $N$ atal (SES) não possui a informação.

CTA/C oas O linda (SMS) possui a informação, mas não a liberou em tempo hábil para a pesquisa. 
Como se constata, a maioria dos que fizeram o teste nos CTA/Coas/ NE pertence à categoria de heterossexuais (73,2\%). Os homossexuais representaram $7,2 \%$ dos testados e os bissexuai s constituíram 5,3\%. Essa mesma ordenação das proporções é vista nos diferentes CTA/ Coas, exceto em Imperatriz, São Luís, Teresina e Fortaleza (SMS), onde o grupo dos bissexuais apresenta um percentual mais el evado do que o da categoria homossexual.

Os heterossexuais apresentaram maior proporção de testes positivos $(37,4 \%)$, seguidos dos homossexuais $(25,2 \%)$ e dos bissexuais $(15,8 \%)$. Esta seqüência segue a freqüência com que os indivíduos pertencentes a essas categorias de exposição procuraram os CTA/ Coas, conforme ficou constatado na Tabela 3.

Analisou-se o grau de positividade dos testes dentro de cada categoria, por ser um indicador que elimina o viés das variadas freqüências de testes realizados em cada uma delas. Segundo os dados mostrados na Tabela 4, a proporção de testes positivos é maior entre os usuários de drogas endovenosas (17,6\%), seguidos dos homossexuais (11\%), bissexuais (10,9\%) e dos transfundidos (5,3\%). Entre os heterossexuais, a positividade foi de apenas $1,6 \%$, sendo o mais baixo percentual observado em todos os CTA/ Coas que informaram esse dado. Contudo, esses dados devem ser relativizados, uma vez que estas informações não estavam disponíveis para seis dos 13 CTA/ Coas que podiam fornecê-la.

Investigou-se a faixa de idade em que os testes positivos eram mais freqüentes, como consta na Tabela 5, onde se verifica que cerca de $75 \%$ dos testes positivos concentram-se nas faixas etárias entre 20 e 39 anos. Esse comportamento pode ser explicado pelo fato de essas faixas etárias terem sido as que mais freqüentemente procuraram os CTA/Coas/NE.

A importância do anonimato para o usuário foi avaliada com base na informação constante nos questionários utilizados pel os CTA/ Coas, observando-se que este (uma das principais normas adotadas pel os CTA/Coas) não é considerado pelo usuário como um fator determinante na busca pelo serviço. A proporção de $68 \%$ respondeu que viria ao CTA/Coas mesmo que ele não fosse anônimo, o que parece indicar que os usuários estão mais preocupados em ter um serviço ao qual possam recorrer, do que com o fato de serem identificados. Entretanto, não se pode ignorar a importância de garantir a possibilidade de realização da testagem anônima aos $11 \%$ dos usuários que afirmaram que não procurariam o CTA/ Coas caso tivessem que revelar sua identidade.
Foi ainda calculada a proporção de usuários soropositivos para o HIV identificados pelos CTA/ Coas em relação ao total dos identificados no município, no primeiro semestre de 1996. Apenas três CTA/ Coas informaram esta questão. No CTA/ Coas de Fortaleza (SES), foram identificados 19,1\% (40/209) dos positivos do município. Em Recife essa proporção foi de 13,1\% (40/306), e, em Arapiraca, nenhum dos três usuários com resultado positivo para o HIV no município foram identificados pelo CTA/Coas.

No que se refere à mudança de comportamento provocada pelo aten dimento, nenhum CTA/Coas possui qualquer instrumento ou forma de mensuração acerca da mudança de comportamento almejada. Não se consegue uma aferição consistente quanto ao efeito dos aconselhamentos e das medidas preventivas que se busca incentivar no usuário. Nesta pesquisa, ficou claro que o conhecimento da equipe em relação a essa questão é puramente intuitivo e empírico. Houve alguns depoimentos quanto à probabilidade de que alguma mudança tenha ocorrido no comportamento do usuário, em razão da sua assi duidade na busca mensal dos preservativos. Este ponto merece uma reflexão. Não prevista originalmente no Projeto CTA/Coas, a distribuição de preservativos passou a funcionar como uma estratégia que garante a adesão da clientela e tem sido avaliada positivamente pelas equipes e usuários. Um usuário que vem ao CTA/ Coas buscar seus preservativos freqüentemente traz amigos e parentes para fazerem o teste. A maioria deles referiu não fazer objeção a comparecer aos CTA/Coas para buscar preservativos, mesmo que possam vir a ser identificados. Mais curioso é que a distribuição dos preservativos tem si do o único indício de alguma prevenção, se bem que, de acordo com os depoimentos dos profissionais, o fato de pegar os preservativos não significa que os mesmos estejam sendo usados como se preconiza.

O uso de preservativo (como indicador informal de mudança de comportamento) também pode ser detectado pelo depoimento de alguns usuários ao retornarem ao CTA/ Coas. Todavia, os próprios técnicos têm consciência da dificuldade em avaliar esse tipo de informação, uma vez que, para eles, mudança de comportamento é algo bem mais amplo e complexo do que a simples referência do usuário de estar utilizando camisinha. Além disso, é difícil isolar o que dessa mudança se deveu ao aconselhamento e o que foi influenciado por outros fatores. 
Proporção de testes positivos por categorias de exposição em relação ao total de testes realizados em cada catego ria (freqüência intracategoria). Centros de Testagem Anônima/Centro de O rientação e Apoio Sorológico da região Nordeste, Brasil.

\begin{tabular}{|c|c|c|c|c|c|c|c|c|}
\hline & & & & Categorias 0 & e exposição & & & \\
\hline & Perinatal & Transfundido & Hemofílico & $\begin{array}{l}\text { Usuário } \\
\text { de droga } \\
\text { endovenosa }\end{array}$ & Homossexual & Bissexual & Heterossexual & $\begin{array}{l}\text { Sem } \\
\text { informação }\end{array}$ \\
\hline Imperatriz (SES*) & - & - & - & 33,3 & - & 25,0 & 4,4 & - \\
\hline São Luís (SM S**) & - & 37,5 & - & 50,0 & 12,9 & 8,2 & 2,5 & 4,8 \\
\hline Fortaleza (SES) & - & - & - & - & 13,0 & - & 1,8 & - \\
\hline Fortaleza (SM S) & - & - & - & - & 16,0 & 5,9 & - & - \\
\hline Recife (SMS) & - & - & - & - & 13,1 & 22,6 & 1,3 & 1,9 \\
\hline Aracaju (SES) & - & - & - & 10,0 & 10,5 & 3,7 & 1,2 & - \\
\hline Maceió (SMS) & - & - & - & - & - & 2,6 & 1,0 & - \\
\hline Total & - & 5,3 & - & 17,6 & 11,0 & 10,9 & 1,6 & 3,3 \\
\hline
\end{tabular}

* Secretaria Estadual de Saúde; ** Secretaria M unicipal de Saúde.

O bs.: CTA/Coas Teresina (SES), M aceió (SES) e Salvador (SES) não informaram.

CTA/Coas Natal (SES) e Arapiraca (SMS) não possuem a informação.

CTA/Coas Olinda (SMS) possui a informação, mas não a liberou em tempo hábil para a pesquisa.

Proporção de testes positivos segundo faixas etárias. Centros de Testagem Anônima/Centro de O rientação e Apoio Sorológico da região Nordeste, Brasil.

\begin{tabular}{|c|c|c|c|c|c|c|c|c|c|}
\hline & \multicolumn{9}{|c|}{ Faixas etárias } \\
\hline & $<15$ anos & 15 a 19 & 20 a 24 & 25 a 29 & 30 a 34 & 35 a 39 & 40 a 44 & 45 a 49 & 50 e mais anos \\
\hline Imperatriz (SES*) & - & 8,3 & 16,7 & 25,0 & - & 25,0 & 16,7 & - & 8,3 \\
\hline São Luís (SM S**) & 5,6 & - & 16,7 & 14,8 & 14,8 & 24,0 & 11,1 & 9,3 & 3,7 \\
\hline Teresina (SES) & 5,0 & 2,5 & 20,0 & 20,0 & 20,0 & 15,0 & 7,5 & 5,0 & 5,0 \\
\hline Fortaleza (SES) & - & 5,0 & 12,5 & 22,5 & 25,0 & 22,5 & 7,5 & 5,0 & - \\
\hline Fortaleza (SMS) & - & 16,7 & - & 16,7 & 33,2 & - & 16,7 & - & 16,7 \\
\hline Recife (SM S) & - & 10,0 & 7,5 & 30,0 & 27,5 & 12,5 & 2,5 & 5,0 & 5,0 \\
\hline Aracaju (SES) & - & - & 15,4 & 15,4 & 7,7 & 38,4 & - & 7,7 & 15,4 \\
\hline Total & 2,4 & 4,4 & 14,2 & 21,0 & 19,5 & 20,0 & 7,8 & 5,8 & 4,9 \\
\hline
\end{tabular}

* Secretaria Estadual de Saúde; ** Secretaria Municipal de Saúde.

o bs.: CTA/Coas Natal (SES) e Maceió (SES) não informaram.

CTA/Coas M aceió (SMS) e Salvador (SES) informaram outras faixas etárias.

CTA/C oas O linda (SMS) possui a informação, mas não a liberou em tempo hábil para a pesquisa.

CTA/Coas Arapiraca (SMS) não possui a informação.

\section{Discussão}

Considerando-se as heterogenei dades dos 17 CTA/Coas e a necessi dade de superação das dificuldades e dos limites de cada um, a equipe avaliadora, juntamente com um representante de cada unidade, definiu critérios de excelência para um CTA/Coas: possuir bom relacionamento com os programas locais, estadual e nacional de DST/ Aids; estar articulado à rede de referência e contra-referência para os portado- res de HIV/ Aids; manter sistema de divulgação; possuir boa articulação com laboratório de referência; ter comprometimento com os princípios de saúde pública; possuir coordenação própria; dispor de equipe multidisciplinar; ter respaldo institucional; manter a rotina de atendimento oferecendo uma testagem rápida, confiável e aconselhamento individual e coletivo prée pós-teste; oferecer boas condições de acesso; garantir o anonimato; efetuar o registro sistemático das atividades. Tendo como base 
tais critérios, toda a equipe avaliadora, em consenso, concluiu que nenhum dos serviços investigados poderia ser classificado como excelente. Os limites e as dificuldades apontados nos itens anteriores mostraram-se como os principais fatores restritivos dessa excelência.

O processo de avaliação integrada, desenvolvido nesta pesquisa, gerou transformações e suscitou expectativas que terão continuidade para além da finalização da mesma. A mobilização dos profissionais dos CTA/Coas, advinda da consciência da necessidade de se auto-avaliarem, foi um ponto positivo e contribuiu em muito para a adesão dos mesmos à pesquisa. $A$ falta de integração da rede e o isolamento de vários CTA/Coas pôde ser dirimida nos encontros coletivos durante o processo. Esses encontros contribuíram para a reflexão acerca das diversas práticas, para a identificação dos profissionais e para o estreitamento de suas relações. Do treinamento até a entrega dos relatórios das equipes, os CTA/Coas tiveram a primeira oportunidade de se auto-avaliar sob diferentes aspectos, de consolidar seus dados, de adotar novos procedimentos em suas rotinas, de olhar para si e apontar os problemas que enfrentam e as propostas de solução para os mesmos.

É preciso ressaltar que uma avaliação possui uma historicidade; portanto, os problemas e dificuldades identificados, bem como as virtudes e pontos positivos constatados, caracterizam-se como fatores dinâmicos, que variam de acordo com mudanças nas condições que determinam seu aparecimento ou sua superação.

As conclusões que a equipe do Claves pôde extrair da avaliação foram submetidas aos profissionais dos CTA/ Coas estudados, que acrescentaram a elas as suas próprias sugestões. No entanto, é preciso destacar que as conclusões aqui apresentadas refletem a dinâmica de um processo provisório, que pode e deve transcender as deficiências, ressaltar as qualidades e va-
Iorizar os profissionais que realizam esse serviço de al ta relevância social no campo da saúde, validado pelos que dele usufruem.

A avaliação propiciou espaço para se concluir que, do ponto de vista dos gestores e mesmo por parte dos outros profissionais de saúde, os CTA/ Coas não ocupam lugar destacado no sistema. Sua articulação com os serviços de saúde é distanciada, não são reconhecidos pela rede pública eàs vezes seus profissionais são estigmatizados. Dentro da própria área de atuação dos serviços das DST/Aids, os CTA/ Coas não estabelecem um contato profissional contínuo com seus pares.

Vale destacar a novidade desses centros, que ultrapassam na sua concepção e prática modelos reducionistas de organização do atendimento, quando introduzem: a) a multiprofissionalidade como fundamento das equipes, estabelecendo as bases de relações horizontais e comunicativas; b) uma nova prática de educação e saúde, onde se articulam informação, comunicação e inter-relação direta com os usuários, tratados enquanto pessoas e membros de uma comunidade familiar, de gênero, de opção sexual e inseridos em uma realidade sócio-cultural; c) uma nova imagem de serviço público, on de os interesses e necessidades dos clientes são atendidos dinamicamente, dentro de princípios éticos (e não burocráticos) estabelecidos.

Tais valores e lições aprendidas, nascidas do cotidiano, alimentam-se da valorização e da crença no serviço público e na construção dos atores envolvidos; enaltecem e dão aos CTA/ Coas um lugar de sinalizador no campo da prática e na reflexão epistemológica, ultrapassando o nível da realização empírica. Esse valor, ressaltado na avaliação apresentada, independe das situações ideais ou problemáticas em que as ações dos CTA/Coas se desenvolvem na macrorregião Nordeste.

\section{Agradecimentos}

Aos técnicos dos CTA/Coas, em especial, pela colaboração e participação no processo da avaliação. A Kathie Njaine, Jussara Garcia Cel estino e Marcelo Rasga Moreira, pela participação no trabalho de campo. E a Marcelo da Cunha Pereira, pelo apoio em informática.

Agradecimentos também ao subsídio dado à pesquisa pela CN-DST/Aids. 


\section{Referências}

ANDRÉ, M. E. D. A., 1982. Texto, contexto e significados: al gumas questões na análise de dados qualitativos. Cadernos de Pesquisa, 45:66-71.

BRASIL. Ministério da Saúde/ Abrasco/Claves, 1996. Avaliação dos Centros de Orientação e Apoio Sorológico-CTA/Coas da Região Nordeste. Relatório Final de Pesquisa. Rio de Janeiro: Ministério da Saúde/ Abrasco/Claves.

DENZIN, N. K., 1970. The Research Act. Chicago: Aldine Publishing Co.

DONABEDIAN, A., 1980. Explorations in Quality Assessment and Monitoring:The Definition of Quality and Approaches to its Assessment. Michigan: Ann Harbor/Health Administration Press.

HABERMAS, J., 1987. Teoria de la Acción Comunicativa. Madrid: Taurus.

JICK, T. D., 1979. Mixing quantitative and qualitative methods: triangulation and action. AdministrativeScience Quaterly, 24:50-72.

KANT, I., 1980. Lógica transcendental. In: Crítica à Razão Pura (V. Rodhen \& V. B. Moosburger, org.), pp. 56-99, São Paulo: Editora Abril.
MINAYO, M. C. S., 1992. O Desafio do Conhecimento. São Paulo: Editora Hucitec.

MINAYO, M. C. S. \& SANCHES, O., 1993. Quantitativo-qualitativo: oposição ou complementaridade. Cadernos de Saúde Pública, 9:239-262.

SAM AJA, J., 1992. La combinación de métodos: pasos para una comprensión dialectica del trabajo interdisciplinario. Educación Médica y Salud, 26:434.

SCHRIMSHAW, S. E. \& HURTADO, E., 1987. Rapid Assessment Procedures for Nutrition and Primary Health Care. Anthropological Approaches to Improving Programs Effectiveness. Tokio: The United Nations University.

SHUTZ, A., 1982. A commonsense and scientific interpretation of human actions. In: Collected Papers II (A. Brodersen, ed.), 2a ed., pp. 20-33, The Hague: Martinus Nijhoff. 